\title{
On the Conceptualization of Total Disturbance and its Profound Implications
}

\author{
Sen Chen \\ Chinese Academy of Sciences
}

Wenyan Bai

Beijing Aerospace Automatic Control Institute

Yu Hu

Cleveland State University

Yi Huang

Chinese Academy of Sciences

Wenbing Zhao

Cleveland State University, w.zhao1@csuohio.edu

Follow this and additional works at: https://engagedscholarship.csuohio.edu/enece_facpub

Part of the Electrical and Computer Engineering Commons

How does access to this work benefit you? Let us know!

Publisher's Statement

The final publication is available at Springer via http://dx.doi.org/10.1007/s11661-004-0269-3

\section{Repository Citation}

Chen, Sen; Bai, Wenyan; Hu, Yu; Huang, Yi; and Zhao, Wenbing, "On the Conceptualization of Total Disturbance and its Profound Implications" (2020). Electrical Engineering \& Computer Science Faculty Publications. 453.

https://engagedscholarship.csuohio.edu/enece_facpub/453

This Letter to the Editor is brought to you for free and open access by the Electrical Engineering \& Computer Science Department at EngagedScholarship@CSU. It has been accepted for inclusion in Electrical Engineering \& Computer Science Faculty Publications by an authorized administrator of EngagedScholarship@CSU. For more information, please contact library.es@csuohio.edu. 


\title{
On the conceptualization of total disturbance and its profound implications
}

\author{
Sen CHEN, Wenyan BAI, Yu HU, Yi HUANG \& Zhiqiang GAO \\ Citation Chen S, Bai W Y, Hu Y, et al. On the conceptualization of total disturbance and its profound implica- \\ tions. Sci China Inf Sci, 2020, 63(2): 129201, https://doi.org/10.1007/s11432-018-9644-3
}

Dear editor,

How to deal with uncertainties and/or disturbances is a central issue pushing the development of both control science and control technology. Among various approaches, the active disturbance rejection control (ADRC) has been successfully implemented in various industrial practices because of its uniqueness in concepts, simplicity in engineering implementation, and superior performance. The ideology of ADRC was reviewed in $[1,2]$, which insightfully proposed the integrator chain of the controlled variable as the most fundamental structure for control systems, linear or nonlinear, and went further to creatively generalize the concept of disturbance to the "total disturbance".

In many literatures, ADRC is usually illuminated by the control problem of nonlinear uncertain systems in the integrator chain form with the total disturbance appearing in the same channel as that of the control input (see Appendix B for a detailed description). Hence, some doubts about the capability of ADRC are raised. (1) Can ADRC handle uncertain systems in a more general form? (2) Can ADRC tackle mismatched uncertainties and/or disturbances?

These doubts are caused by a misunderstanding of the crucial concepts of the integrator chain form and the "total disturbance" in ADRC. This miss- ing link motivates this work. By systematically studying the control problem for systems with multiple disturbances, this study shows that it is usually neither possible nor necessary to deal with each disturbance individually. Via the profound implications of the integrator chain and the total disturbance, the capability of ADRC is explored for more generic uncertain systems with multiple disturbances, internal and external, which are unobservable and/or mismatched. Furthermore, the essences of the integrator chain form and the "total disturbance" are revealed as follows. (1) The integrator chain from the control input to the controlled variable is the kernel of most dynamical systems associated with control engineering practice, rather than being a simple special case. (2) The total disturbance corresponds to the difference between the dynamics of the physical plant and this integrator chain. It is the lumped effects of all disturbances, both internal and external, projected at the control input side. Finally, experiments on a two-mass-spring (TMS) system illustrate the capability of the proposed ADRC design for multiple disturbances. See Appendix $\mathrm{C}$ for the details of this study's main contributions.

Problem description. Consider the following class of nonlinear uncertain systems:

$$
\left\{\begin{array}{l}
\dot{x}(t)=A x(t)+b_{u} u(t)+B_{f} f(x(t), u(t), t), \\
y(t)=c^{\mathrm{T}} x(t), \quad t \geqslant t_{0},
\end{array}\right.
$$


where $x(t) \in \mathbb{R}^{n}$ is the state vector, $y(t) \in \mathbb{R}$ is the measured output to be controlled, $u(t) \in \mathbb{R}$ is the control input and $f(x, u, t)=\left[f_{1} \cdots f_{p}\right]^{\mathrm{T}} \in$ $\mathbb{R}^{p}$ represents multiple disturbances, which include external disturbances, unmodeled dynamics and parametric perturbations. The matrices $A \in \mathbb{R}^{n \times n}, B_{f} \in \mathbb{R}^{n \times p}, b_{u} \in \mathbb{R}^{n \times 1}$ and $c \in \mathbb{R}^{n \times 1}$ are known, and $t_{0}$ is the initial time. Without loss of generality, $b_{u}, B_{f}$, and $c$ are assumed to be nonzero, and $B_{f}$ is column full rank.

The model (1) can describe a variety of practical systems, such as the TMS system, which represents a typical vibration system in practice (see Appendix D for a detailed description of TMS systems).

Consider the disturbances $f$ in a function set $\Omega_{f}$ which contains $f(x, u, t) \equiv \mathbf{0}$. The control objective for the uncertain system (1) is to design a control input $u(t)$ such that, for any $f \in \Omega_{f}$, the output $y(t)$ can track the bounded reference signal $r(t)$ with bounded derivatives $r^{(i)}(t)(i \geqslant 1)$.

Main results. The study of the control problem for systems with multiple disturbances (1) shows that tackling multiple disturbances individually seems impossible because the disturbances might be unobservable and mismatched (see Appendix E for a detailed discussion). However, ADRC provides a different ideology of handling disturbances that focuses on the integrator chain from the control input to the controlled variable and seizes the total disturbance, which is the difference between the real physical plant and the ideal integrator chain.

Next, the integrator chain and the total disturbance of the uncertain system (1) will be analyzed.

Assume that the uncertain system (1) satisfies the following assumption.

Assumption 1. For every $f \in \Omega_{f}$, the relative degree from $u$ to $y$ is $n$.

The relative degree is the minimum number of integrators from the control input to the controlled output, which can be definitely determined by the control mechanism of the physical plant, regardless of the model description.

The controlled variable $y$ and its up to $(n-1)$ th derivatives are denoted as a new state vector $\tilde{x}(t)=\left[\begin{array}{lll}\tilde{x}_{1}(t) & \cdots & \tilde{x}_{n}(t)\end{array}\right]^{\mathrm{T}} \triangleq\left[y(t) \cdots y^{(n-1)}(t)\right]^{\mathrm{T}}$. The following theorem describes the relationship between $\tilde{x}$ and $x$, and further explores the connection between the control input $u$ and the new state $\tilde{x}$.

Theorem 1. If the uncertain system (1) satisfies Assumption 1, then

(1) For all $x_{0} \in \mathbb{R}^{n}$, there exist a neighborhood of $x_{0}, U\left(x_{0}\right)$, and a function $g_{f, x_{0}}(\tilde{x}, t)$ dependent on $\left(f, x_{0}\right)$, such that $x=g_{f, x_{0}}(\tilde{x}, t)$ for $x \in U\left(x_{0}\right)$;

(2) For $x \in U\left(x_{0}\right)$, the integrator chain form of the uncertain system (1) is

$$
\left\{\begin{array}{l}
\dot{\tilde{x}}_{i}(t)=\tilde{x}_{i+1}(t), \quad 1 \leqslant i \leqslant n-1, \\
\dot{\tilde{x}}_{n}(t)=c^{\mathrm{T}} A^{n-1} b_{u} u(t)+c^{\mathrm{T}} A^{n} g_{f, x_{0}}(\tilde{x}, t) \\
\quad+\sum_{k=0}^{n-1} c^{\mathrm{T}} A^{n-k-1} B_{f} f^{(k)}\left(g_{f, x_{0}}(\tilde{x}, t), u, t\right), \\
y(t)=\tilde{x}_{1}(t) .
\end{array}\right.
$$

The integrator chain form (2) reveals that the only known information about the uncertain system (1) is $c^{\mathrm{T}} A^{n-1} b_{u} u$ and the rest is equivalent to the total influence of the multiple disturbances $f$ on the controlled variable. Conceptualize this equivalent total effect of the multiple disturbances $f$ on the controlled variable as the total disturbance

$$
f_{\text {total }} \triangleq c^{\mathrm{T}} A^{n} g_{f, x_{0}}(\tilde{x}, t)+\sum_{k=0}^{n-1} Q_{k}\left(g_{f, x_{0}}(\tilde{x}, t), u, t\right),
$$

where

$$
Q_{k}(x, u, t) \triangleq\left\{\begin{array}{lr}
0, & \text { if } c^{\mathrm{T}} A^{n-k-1} B_{f}=0, \\
c^{\mathrm{T}} A^{n-k-1} B_{f} f^{(k)}(x, u, t), & \text { else. }
\end{array}\right.
$$

Then the following theorem illuminates the significance of the conceptualization of total disturbance.

Theorem 2. Consider the uncertain system (1) with Assumption 1. Its total disturbance $f_{\text {total }}(3)$ is both observable and matched.

Although the multiple disturbances $f$ might be unobservable and mismatched, the "total disturbance" for the uncertain system (1), $f_{\text {total }}$, is not only observable but also matched. Thus, to realize the satisfied tracking performance despite the multiple disturbances, it only needs to deal with the total disturbance $f_{\text {total }}$, which is not necessarily a certain concrete disturbance.

To estimate the total disturbance $f_{\text {total }}$, a commonly designed extended state observer (ESO) [2], which corresponds to the integrator chain form (2), is presented as follows:

$$
\begin{aligned}
{\left[\begin{array}{c}
\dot{\tilde{\tilde{x}}}(t) \\
\dot{\hat{f}}_{\text {total }}(t)
\end{array}\right]=} & {\left[\begin{array}{cccc}
0 & 1 & \cdots & 0 \\
\vdots & 0 & \ddots & \vdots \\
\vdots & \vdots & \ddots & \\
0 & 0 & \cdots & 0
\end{array}\right]\left[\begin{array}{c}
\hat{\tilde{x}}(t) \\
\hat{f}_{\text {total }}(t)
\end{array}\right]+\left[\begin{array}{c}
0 \\
\vdots \\
0 \\
1 \\
0
\end{array}\right] u } \\
& +l_{\text {ESO }}\left[\begin{array}{c}
1 \\
0 \\
\vdots \\
0
\end{array}\right]^{\mathrm{T}}(\tilde{x}(t)-\hat{\tilde{x}}(t))
\end{aligned}
$$

where $\hat{\tilde{x}} \in \mathbb{R}^{n}$ and $\hat{f}_{\text {total }} \in \mathbb{R}$ are expected to be the online estimations of the up to $(n-1)$ th order derivatives of the controlled variable $\tilde{x}(t)$ and 
the total disturbance $f_{\text {total }}$, respectively. To simplify the tuning of the ESO's parameter, $l_{\mathrm{ESO}}$ is designed as

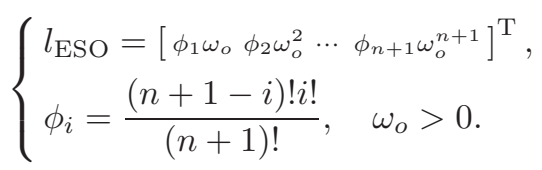

By utilizing the estimation from the ESO (4), an ADRC law can be designed as follows:

$$
u(t)=\left\{\begin{array}{l}
0, \quad t_{0} \leqslant t<\tilde{t}_{0}, \\
\left(-\sum_{i=1}^{n} k_{c, i}\left(\hat{\tilde{x}}_{i}(t)-r^{(i-1)}(t)\right)-\hat{f}_{\text {total }}(t)\right. \\
\left.+r^{(n)}(t)\right) /\left(c^{\mathrm{T}} A^{n-1} b_{u}\right), t \geqslant \tilde{t}_{0},
\end{array}\right.
$$

where the feedback gain $k_{c}=\left[\begin{array}{llll}k_{c, 1} & k_{c, 2} & \cdots & k_{c, n}\end{array}\right]^{\mathrm{T}}$ is chosen such that $A_{k_{c}} \triangleq\left[\begin{array}{cccc}0 & 1 & \cdots & 0 \\ \vdots & \ddots & \ddots & \vdots \\ 0 & \cdots & 0 & \vdots \\ k_{c, 1} & k_{c, 2} & \cdots & k_{c, n}\end{array}\right]$ is a Hurwitz matrix. Details about $\tilde{t}_{0}$, which represents the time after which the peaking of the ESO (4) ends, are presented in Appendix F.2.

Consider the following rational assumption.

Assumption 2. (i) $f(x, u, t)$ is smooth for $(x, u) \in \mathbb{R}^{n+1}$ and piecewise smooth for $t \in$ $\left(t_{0}, \tilde{t}_{1}\right) \cup \bigcup_{i \geqslant 1}\left(\tilde{t}_{i}, \tilde{t}_{i+1}\right)$. There exists a positive $\varphi_{d}$ such that $\min _{i \geqslant 1}\left\{\left|\tilde{t}_{i+1}-\tilde{t}_{i}\right|,\left|\tilde{t}_{1}-t_{0}\right|\right\} \geqslant \varphi_{d}$. (ii) $g_{f, x_{0}}(\tilde{x}, t)$ and its partial derivatives with respect to $\tilde{x}$ and $t$ are bounded by a continuous function $\psi_{1}(\tilde{x})$ for $\left(f, x_{0}, t\right) \in \Omega_{f} \times \mathbb{R}^{n} \times$ $\left(\left(t_{0}, \tilde{t}_{1}\right) \cup \bigcup_{i \geqslant 1}\left(\tilde{t}_{i}, \tilde{t}_{i+1}\right)\right)$. (iii) $Q_{k}(x, u, t)(0 \leqslant k \leqslant$ $n-1)$ and its partial derivatives with respect to $x$ and $t$ are bounded by a continuous function $\psi_{2, k}(x, u)$ for $t \in\left(t_{0}, \tilde{t}_{1}\right) \cup \bigcup_{i \geqslant 1}\left(\tilde{t}_{i}, \tilde{t}_{i+1}\right)$. (iv) There exist positive constants $\varphi_{1}$ and $\varphi_{2}$ such that $\left(\frac{\partial f_{\text {total }}}{\partial u}+\bar{b}\right) / \bar{b} \in\left[\varphi_{1}, \varphi_{2}\right] \subset\left(0,2+\frac{2}{n}\right)$ for $t \geqslant t_{0}$.

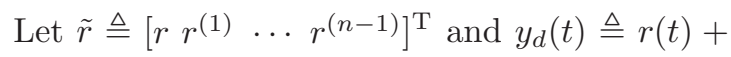
$\left[\begin{array}{llll}1 & 0 & \cdots & 0\end{array}\right] \mathrm{e}^{A_{k_{c}}\left(t-t_{0}\right)}\left(\tilde{x}\left(t_{0}\right)-\tilde{r}\left(t_{0}\right)\right)$. Then, the following theorem illuminates the capability of the ADRC design (4)-(6) for handling multiple disturbances of the uncertain system (1).

Theorem 3. Consider the system (1) with the ADRC design (4)-(6). Assume that Assumptions 1 and 2 are satisfied. There exist positives $\omega^{*}$ and $\eta_{i}^{*}(1 \leqslant i \leqslant 3)$ which depend on $\left(\tilde{x}\left(t_{0}\right), \psi ., \varphi ., k_{c}\right)$, such that the closed-loop system has the following properties for all $\omega_{0} \geqslant \omega^{*}$ :

$$
\begin{array}{r}
\sup _{t \in\left[t_{0}, \infty\right)}\left|y(t)-y_{d}(t)\right| \leqslant \eta_{1}^{*} \max \left\{\frac{\ln \omega_{o}}{\omega_{o}}, \frac{1}{\omega_{o}}\right\}, \\
\left\|\left[\begin{array}{c}
\tilde{x}(t)-\hat{\tilde{x}}(t) \\
f_{\text {total }}-\hat{f}_{\text {total }}(t)
\end{array}\right]\right\| \leqslant \frac{\eta_{2}^{*}}{\omega_{o}}+\eta_{2}^{*} \mathrm{e}^{-\eta_{3}^{*} \omega_{o}\left(t-\tilde{t}_{i}\right)}, \\
t \in\left[\tilde{t}_{i}, \tilde{t}_{i+1}\right), i \geqslant 0 .
\end{array}
$$

The result (7) indicates that the tracking error $\left|y(t)-y_{d}(t)\right|$ is bounded, and, more importantly, tunable by the ESO's bandwidth $\omega_{o}$, where $y_{d}(t)$ is the desired trajectory exponentially converging to the reference signal $r(t)$. Moreover, Eq. (8) illustrates that the estimation errors for the derivatives of the controlled variable and the total disturbance are also bounded in each smooth region, and tunable by the ESO's bandwidth $\omega_{o}$. Hence, Theorem 3 demonstrates that a satisfactory transient performance of both the tracking and estimating can be achieved via the ADRC design (4)-(6).

The full discussion on the ADRC design via the conceptualization of total disturbance and the proofs of Theorems 1-3 are presented in Appendix F. Additionally, some extended discussion on ADRC designs are shown in Appendix G. Furthermore, the experimental verification on a TMS system, which demonstrates the capability of ADRC to handle multiple uncertainties, is presented in Appendix H.

Conclusion. This study reveals the profound implications of the integrator chain and the "total disturbance". The integrator chain is the essential dynamics from the control input to the controlled variable, rather than a simple and special case. This essential structure ensures that the "total disturbance", which corresponds to the difference between the ideal integrator chain and the real physical plant, is not only observable but also matched. More importantly, the "total disturbance" is the equivalent total effect caused by multiple disturbances, both internal and external. Thus, even if some specific disturbances are unobservable and/or mismatched, the keys to control systems with multiple disturbance are to seize the integrator chain from the control input to the controlled variable, and then to handle the total disturbance. This is what ADRC has done.

Acknowledgements This work was supported by $\mathrm{Na}$ tional Center for Mathematics and Interdisciplinary Sciences, Chinese Academy of Sciences.

Supporting information Appendixes A-H. The supporting information is available online at info.scichina.com and link.springer.com. The supporting materials are published as submitted, without typesetting or editing. The responsibility for scientific accuracy and content remains entirely with the authors.

\section{References}

1 Han J. From PID to active disturbance rejection control. IEEE Trans Ind Electron, 2009, 56: 900-906

2 Huang Y, Xue W. Active disturbance rejection control: methodology and theoretical analysis. ISA Trans, 2014, 53: 963-976 